\title{
A retrospective view on the history of natural sciences in $\mathbf{X X - X X I}$
}

\author{
Vladislav Sergeyevich Olkhovsky \\ Institute for Nuclear Research, Kiev, Ukraine; olkhovsky@mail.ru \\ Received 1 December 2009; revised 8 January 2010; accepted 30 January 2010.
}

\begin{abstract}
The presented paper is dedicated to a new retrospective view on the history of natural sciences in XX-XXI cc, partially including the science philosophy (mainly, the problems of the scientific realism, i.e. the correspondence of science to reality) and also a novel scheme for different classes of sciences with different objects and paradigms. There are analyzed the chosen "great" and "grand" problems of physics (including the comprehension of quantum mechanics, with a recently elaborated new chapter, connected with time as a quantum observable and time analysis of quantum processes) and also of natural sciences as a whole. The particular attention is paid to the interpretation questions and slightly to the aspects, inevitably connected with the world- views of the researchers (which do often constitute a part of the interpretation questions).
\end{abstract}

Keywords: science history; science realism; paradigm; problem of interpretation and comprehension of quantum mechanics; the wave-function collapse; the Einstein-Podolsky-Rosen paradox; time as a quantum observable, canonically conjugated to energy; maximal hermitian time operator; time analysis of quantum processes; relationship between physics and biology; problem of origin of biologic life; reductionism; cosmologic problem; Big Bang; anthropic principle

\section{INTRODUCTION}

In the science history and in the science philosophy of XX-XXI (especially in the field of the natural sciences and most of all in physics) there has been a lot of interesting things, which had not obtained a sufficiently complete elucidation and analysis yet. Firstly, under the influence of scientific and technological progress a great attention has been paid to the development of such di- rection in the science philosophy as the scientific realism (i.e. the correspondence of the science to the reality), which has successively acquired three forms: the naive realism, the usual realism and the critical science realism. Secondly, some new important problems of physics (especially the problem of the essentially probabilistic description of the reality of the microscopic world, the problem of the essential influence of the observer on the reality, the collapse of the wave function and the Einstein-Podolsky-Rosen paradox) had been revealed in the development of quantum mechanics; the continuously complicated explanation of the Universe origin and the expansion after the Big Bang; and no succeeded attempt in explaining the origin of the biological life in terms of physics and other natural sciences, all being with a variety of interpretation versions (often connected with the world-views of the researchers), cause to undertake a new view of the science history. And thirdly, a clear analysis of the variety of known sciences brings to the re-considering of the science classification and a novel scheme for different classes of natural sciences with quite different objects (including not only simple natural phenomena and processes, but also the human intelligent design and the origin of the Universe and life). Finally, a simple study of the enlargement of mathematics in practically all of sciences did not only indicate that mathematics became the branch of the natural sciences (as to the opinion of some scientists, such as N.N.Bogolyubov and others) but has also in fact induced the solution of the long-standing problem of time in quantum mechanics.

\section{THE SCIENTIFIC REALISM AND ITS DIFFERENT KINDS}

If the science does correspond the reality? In the science philosophy the term reality defines the direction, postulating the existence of the reality, independent from the cognitive subject. The scientific realism postulates the existence of the objective truth, the aim of the scientific theories is being declared the revelation of the real truth, the moving force of the scientific progress is declared 
the approach to the truth (the truth is explained as entirely adequate description of the reality). The scientific theories, if they ate really truthful, do describe in an adequate way the reality and the essences, which are postulated by the well tested theories, are existing really.

R.Boyd [1] selected three types of the scientific realism:

The ontological realism assumes that the reality, which is described by the scientific realism, does not depend, on the whole, from our thinking and from the theoretic assumptions. The ontological realism responds to the questions like "what essences are real?", "if the world, which is independent from the observer, does exist?"

The epistemological realism assumes that the scientific theories are confirmed as true and in fact are often approach to the reality. The epistemological realism responds to the question: "is the knowledge about the world possible?"

The semantic realism assumes that "the theoretical terms" of the scientific theories indicate to the realistic essences, i.e. the theories have to be interpreted realistically. The semantic realism responds the question: "if the truth of the objective world is expressed by the scientific language?"

Respectively, the scientific realism on the whole assumes that the scientific theories tend to give the truthful description of the reality which is existing independently ("the truth" signifies here the complete correspondence between the science and the reality). If the scientific theory is really true, then the unobserved essences, which it postulates, are really existing.

A.Bird [2] had formulated the short thesis of the scientific realism. He states that the scientific theories:

a) can be estimated in the terms of the truthfulness or the approach to the truthfulness;

b) their reasonable aim is the truth or the approach to the truth;

c) their success, confirmed by the scientific progress, testimonies their truth;

d) if they are true, then the unobserved essences, which they assume, are really existing;

e) if they are true, they will explain the observed phenomena.

The main argument for the realism is the conclusion on the best explanation of the reality: the scientific realism is the only science philosophy that can explain the scientific progress. The scientific realism is exposed to the critics from the antirealism (antirealism, appeared in the second part of the XX, does represent the scientific philosophy, which is opposite to the realism). Antirealists state that to consider the scientific theories to be true is too risky. Some previous scientific theories were false, for example, the theories of heat matter, phlogiston, the ether conception. So, modern theories can be also false. The position of the scientific realism is criticized by an- tirealists, although it has a lot of supporters.

In the non-uniform current of the scientific realism there are known 3 kinds: naïve, usual and critical.

The naïve realism is the position of the majority of men from the point of view of the common sense. [The common sense is acquired by all normal men during the natural living process, in the overall man communications and in actions with the objects of our usual experience. It is like the assimilation of the natal language with which the common sense is therefore closely connected. In many situations the common sense is used as a matter of fact the primordial universal kind of knowledge]. According to this position, the world is such, which is represented by the modern (however, pre-quantum!) science: those essences, the existence of which are postulated by the well-supported scientific theory, are really existing. And only objects, which are described by the scientific theories, have the authentically real ontological status, and the scientific knowledge as an epistemological base of the science does also represent the realism.

The usual realism is the position of the investigators like $[1,2]$, and also somewhat different positions of a series of other authors (for instance, such as J.Smart, R.Harre, H.Putnam etc.).

Later the more "weak" realistic position appeared the critical realism, with the more modest declarations of its supporters. The critical scientific realism had been declared by the not very equal positions of many various authors. The position of the critical realism had been rather clearly formulated by I.Niiniluotto from the Helsinki University [3], which added some more precise definitions to the position of the critical realism. In particular, he recognizes the conceptual pluralism, under the influence of the uncertainty thesis of W.Quine [4]: our appeal to the world does always occur in some linguistic frame. The thesis of the pessimistic induction forces him to accept the possibility of the untrue theories: the knowledge about the reality is not very trustworthy and demands certain corrections, and even the best scientific theories can contain the mistakes, however the successful theories approach to the reality. The thesis about "the human insertion" signifies that the reality is partially (but only partially!) constructed by the humanity. And in the whole the position of Niiniluotto conserves the realistic optimism: the scientific progress can be rationally explained. As before, the best explanation does consist in that the scientific theories approximate to reveal the truth. Concretely I.Niiniluotto had formulated such thesis of the critical realism [3]:

a) At least, the part of the reality is ontologically independent from the human intelligence.

b) The truth is the semantic relation between the language and the reality.

c) The conception of the truth or the falsification can de used to all linguistic derivatives of the scientific activity including the reports on the observations, the laws 
and the theories. In particular, the statements on the existence have the truthfulness significations.

d) The attainment of the truth is the main aim of the science. The truth is not being found and recognized in a simple way and even the best scientific theories can be false. Nevertheless, it is possible to approach to the truth and obtain the rational conception on the cognitive process.

e) The best explanation of the practical success of the science consists in the approximate truthfulness or successful approaching to the revelation of the truth about the reality. And therefore the scientific progress can be rationally explained.

Not always it is possible clearly distinct the usual scientific realism and the critical scientific realism because in the scientific thinking a lot of attention is paid to the critical analysis of the cognition methods and the scientific knowledge with utilization of all logic cognition methods with understanding of their limitedness. But with the sufficiently completeness of the utilization of the logic cognition methods, the position of the critical realism is better defended against the standard arguments utilized against the realism (usual and especially naïve). If earlier the main attention in the science philosophy had been paid to the justification of the scientific method, during the last dozens of years mainly the questions of the ontological status of objects, introduced by the scientific theories, are discussed. It must be noted that the problems of the quantum theory, revealed as a result of the long (during many years) discussion of N.Bohr with A.Einstein, had seriously undermined the traditional forms of the naïve realism in the science and have strongly influenced not only on physics but also on other kinds of knowledge and on our understanding of the human knowledge at all.

The quantum mechanics, in difference from the classical (non-quantum) physics, revealed that on the microscopic level there is the un-removable indeterminism, represented by the uncertainty relations of Heisenberg, by the essential non-locality of the particle-waves (still unmeasured, i.e. before measurements) and also by the measurements with the discrete interaction of the microscopic objects and the measurement devices (when, for instance, there are the photons are emitted and absorbed). Then in quantum mechanics there is the problem of the interpretation of the quantum measurements and particularly the wave-function collapse etc, when the state of the measured system is formed by the observer [5,6]. All these problems and paradoxes had arisen as a challenge to the philosophy and even now bring to the acute discussions $[5,6]$. And if the majority of the physicists agree with the Bohr Copenhagen interpretation of the quantum mechanics, a certain part of the physicists still assumes that A.Einstein was righteous in his statement that the quantum theory (in its Copenhagen interpretation) does not directly describe the reality. Still the more acute situation had arisen from other quantum phenomena such as the Einstein-Podolsky-Rosen paradox. But as to opinion to some ideologists, such conclusions are justified only in the frame of the physical description and even in such description many of these problems are open even now, so there is no the final necessity now to extrapolate them into the philosophy and theology with profound "revolutionary" philosophical and theological conclusions.

\section{THE DIFFERENT CLASSES OF PARADIGMS IN THE DIFFERENT CLASSES OF NATURAL SCIENCES}

If the objects of natural sciences (physics, chemistry, biology, geology, astronomy etc) are limited by the only natural events and processes, the objects of some other sciences include in their objects also the artificial facts (arte-facts) as creations of the human intelligent design (there are archeology, medicine, criminalistics, and, moreover, mathematics, cybernetics, informatics, and also such humanitarian sciences as history, economics and political sciences). There are also the particular sciences where the origin and history of the Universe or the origin and history of the biological life (including genetics) are studied and where side by side with the scientific method a metaphysical worldview approach of the investigators does, almost inevitably, also take place: Due to the cardinal separation of the investigators because of the incompatibility of their worldviews as to the problems of the origin, the dilemma of the following choice had appeared: either 1) the self-organization of the matter from the null or a less organized level into the much more organized level by virtue of a certain irrational chance or by virtue of unknown now synergetic processes (or phase transitions), or 2) the origin of the Universe and of the life inside it as a result of the supreme intelligent design of a certain super-human creative basis (or a Creator).

And in these three classes of sciences with the different research objects now there are co-existing for scientific researches three different classes of paradigms (the term "paradigms" had been introduced by T. Kuhn in [7]) which exist inside the sciences of their application: the class of paradigms for research of the laws of functioning of the natural processes; the class of paradigms of introducing (or inserting) the human intelligent design inside the natural processes or in the human activity; and finally the class of paradigms of the research of the mechanisms of the origin of the Universe and the life.

Moreover, the first two classes are now already observed to be sometimes overlapped: for instance, in quantum mechanics it is known that the state of the measured system can be in fact formed by the observer [5], i.e. the human intelligent design can actively influence on the currency of the observed natural processes! And in the third class of sciences, which deals with the origin prob- 
lems, for a long time there are known acute collisions of different worldviews which are sometimes expanding into the second group, including mathematics, informatics and cybernetics. Besides the confusion of the different classes of paradigms, such discussions between the supporters of the different worldviews have sometimes become more acute because of the complete incompatibility of the researcher's worldviews, taking the especially sharp forms between the evolutionists and creationists. Even A. Einstein in his last-life period had participated, at least partially and philosophically, in these discussions (see, for instance, [8]): "Considero le dottrine evoluzionistiche di Darwin, Haeckel, Huxley, come tramontate senza speranza" (in English: "I consider the evolutionism doctrines of Darwin, Haeckel and Huxley as being outgoing without any hope to revive").

As to mathematics, one can note that usually the object of every mathematical discipline or theory is taken as the system of the exactly formulated axioms, and the methodology of mathematics consists in the derivations of the logical conclusions and theorems from the chosen axioms. Previously C.Gauss referred to mathematics as "the Queen of the Sciences" [9]. Later it was observed that certain scientific fields (such as theoretical physics) are mathematics with axioms that are intended to correspond to reality. In any case, mathematics shares much in common with many fields in the physical sciences, notably the exploration of the logical consequences of assumptions. And K. Popper concluded that "most mathematical theories are, like those of physics and biology, hypothetico-deductive: pure mathematics there-fore turns out to be much closer to the natural sciences whose hypotheses are conjectures, than it seemed even recently" [10. Moreover, in XX-XXI some mathematicians consider that mathematics has already become practically a branch of natural sciences (theoretical physics) - see, for instance, [11]. And it is in agreement with the known statement of Galileo: "Il libro della natura è scritto in lingua matematica" (in English: "The book of nature is written by the mathematical language") [12]. Now in the theory of quantum collisions and, in particular, in the theory of the dispersion relations the initial assumptions of these theories do contain, besides the physical principles, also the mathematical principle of certain analytic properties of the $S$-matrix in the complex plane of energies (momenta) [11, the first reference]). Finally, namely mathematics generated the solution of the long-standing problem of time as a quantum observable, canonically conjugated to energy, and self-consistent time analysis of quantum processes.

\section{AS TO "GREAT" AND "GRAND" PROBLEMS OF NATURAL SCIENCES}

There is an extensive introduction in the large number of open problems in many fields of physics, published by the Russian physicist V.Ginzburg in [13], which is rather interesting to study. Inside this large list of open problems of modern physics (and in a certain degree of modern natural sciences), represented by V.Ginzburg repeatedly in Russian editions, some of them are marked him "great" or "grand" problems. Between namely these problems I would like to separate three of them.

a) The problem of interpretation and comprehension of quantum mechanics (even of the non-relativistic quantum theory) remains still topical.

The majority of critics of quantum mechanics are unsatisfied with the probabilistic nature of its predictions. One can add here also the questions and paradoxes of the theory of quantum measurements theory, especially like the wave-function reduction and the Einstein-PodolskymRosen paradox. The appearance of quantum mechanics, and, in particular, the discussion of N.Bohr with A. Einstein (lasting many years), had seriously undermined the traditional forms of the naïve realism in the philosophy of the scientific realism and had strongly influenced (and are continuating to influence) not only on physics but also on other kinds of knowledge in the sense of the dependence of the reality on the observer and, moreover, on our understanding of the human knowledge at all. The problem of the relativistic quantum mechanics and quantum field theory is even much more sharp because of the incompatibility of the main premises of the quantum theory and of the relativity theory.

b) The relationship between physics and biology and, specifically, the problem of reductionism.

The main problem, according to V.Ginzburg, is connected with the explanation of the origin of the biologic life and the origin of the human abstract thinking (but the second one, as to me, is connected not with biology but with the origin of the human spiritual life which is far beyond natural sciences). V.Ginzburg assumes that for a possible explanation of the origin of the biologic life one can naturally imagine a certain jump which is similar to some kind of phase transition (or, may be, certain synergetic process). But there are other points of view too.

c) The cosmological problem (in other words, the problem of the Universe origin).

According to V.Ginzburg, it is also a grand problem, or strictly speaking, a great complex of cosmic problems many of which is also far from the solution.

\section{MORE DETAILED COMMENTS ON THE PROBLEM OF COMPREHEN- SION OF QUANTUM MECHANICS}

Various interpretations of quantum mechanics. Not only philosophers of scientific critical realism, but also up to now a certain part of physicists, beginning from A. Ein- 
stein, D.Bohm, Y.Aharonov and some others, did not agree with the Copenhagen interpretation of quantum mechanics and, moreover, had constructed alternative versions of interpretation (see, for instance, [14-22]).

Einstein never accepted quantum mechanics as a "real" and complete theory, struggling to the end of his life for an interpretation that could comply with relativity without complying with the Heisenberg uncertainty principle. As he once said: "God does not play dice", skeptically referring to the Copenhagen interpretation of quantum mechanics which says there exists no objective physical reality other than that which is revealed through measurement and observation.

In 1935 Einstein, Podolsky, and Rosen were formulated their thought experiment, which had been called the $E P R$ paradox (which is also referred to as the EPRB paradox after Bohm, who improved the formulation of the thought experiment). It draws attention to a phenomenon predicted by quantum mechanics known as quantum entanglement, in which measurements on spatially separated quantum systems can instantaneously influence one another. As a result, quantum mechanics violates a principle formulated by Einstein, known as the principle of locality or local realism, which states that changes, performed on one physical system, should have no immediate effect on another spatially separated system. The principle of locality seems to be persuasive, because, according to relativity, information can never be transmitted faster than the speed of light, or causality would be violated. Any theory, violating causality, would be deeply unsatisfying. However, a detailed analysis of the EPR scenario shows that quantum mechanics violates locality without violating causality, because no information can be transmitted using quantum entanglement.

Nevertheless, the principle of locality appeals powerfully to physical intuition, and Einstein, Podolsky and Rosen were unwilling to abandon it. They suggested that quantum mechanics is not a complete theory, just an (admittedly successful) statistical approximation to some yet-undiscovered description of nature. Several such descriptions of quantum mechanics, known as "local hidden variable parameters", were proposed. These deterministically assign definite values to all the physical quantities at all times, and explicitly preserve the principle of locality.

Of the several objections to the then current interpretation of the quantum mechanics spearheaded by Einstein, the EPR paradox was the subtlest and most successful. The EPR paradox has not been resolved or explained, in a way, which agrees with classical intuition, up to this day. It brought a new clarity and permanent shift in thinking about 'what is reality' and what is a 'state of a physical system'.

The shift was caused by the EPR thought experiment, which has shown how to measure the property of a particle, such as a position, without disturbing it. In today's terminology, we would say that they did the determination by measuring the state of a distant but entangled particle. Quantum entanglement is a property of a system of two or more particles (objects) in which the quantum states of the constituting objects are linked together so that one object can no longer be adequately described without full mention of its counterpart - even if the individual objects are spatially separated. According to quantum mechanics, the state of the counterpart particle will instantly change even though we did not disturb it in any local way. It conflicts with our classical intuition with the relativistic principle of locality. Different views on the essence of the quantum entanglement bring to different interpretations of quantum mechanics. The very concept of quantum entanglement also conflicts with our intuition the same way.

However, experiments have shown that entanglement does occur, and in fact quantum entanglement has practical applications in the field of quantum cryptography and quantum computation. Earlier quantum entanglement had been utilized in experiments with quantum teleportation. Quantum teleportation is a technique used to transfer quantum information from one quantum system to another. It does not transport the system itself, nor does it allow communication of information at superluminal (faster than light) speed. Its distinguishing feature is that it can transmit the information present in a quantum superposition, useful for quantum communication and quantum computation. In quantum cryptography, an entangled signal is sent down a communications channel making it impossible to intercept and rebroadcast that signal without leaving a trace. In quantum computation, entangled states allow simultaneous computations to occur in one step.

Entanglement has many applications in quantum information theory [23-31]. Mixed state entanglement can be viewed as a resource for quantum communication. With the aid of entanglement, otherwise impossible tasks may be achieved. Among the best known applications of entanglement there is super-dense coding.

In 1964 J.Bell had shown that many theories, known as hidden variable theories, are either non-local or known as satisfying Bell inequality [16]. Quantum mechanics predicts that this inequality is not satisfied. To make sure, additional experiments were made to confirm that predicted action at distance is indeed instant. Today most physicists agree that local hidden variable theories are untenable and that the principle of locality does not hold. Therefore, the EPR paradox would only be a paradox because our physical intuition does not correspond to physical reality. But even now the topic remains active and some people are still looking for Quantum. quantumquantumquantummechanics is neither "real" (since measurements do not state, but instead prepare properties of the system) nor "local" (since the state vector comprises the simultaneous probability amplitudes for all 
positions), and the properties of entanglement are some of the many reasons why the Copenhagen Interpretation is no longer considered standard by a large proportion of the scientific community. So, the discussion of N.Bohr with A.Einstein had originated so many interesting fundamental results, experimental applications and other (already second or derived) discussions, which have endless continuation up to now, that it was unique in the history of physics.

And now, let us speak some words on the many-world interpretation (MWI) in quantum mechanics (and in quantum cosmology). In this interpretation one assumes the existence of the parallel universes, in every of which the same nature laws and physical constants are acting, but all of them are found in different states. MWI refuses an indeterminate collapse of the wave function which is connected with the measurement in the Copenhagen interpretation. The ideas of MWI had been originated in the phd-thesis of H.Everett bat the term MWI had been proposed by B.S.M. de Witt who had developed that idea, and then the various authors had participated in the further development of that topic [32-41].

In various versions of MWI there two main points: The first one consists in the existence of the wave function for the total Universe, described by the Schroedinger equation, but without any in-determined collapse. The second one consists in that such state of the Universes is the quantum superposition of several (and may be, of the infinite number of) states of the equal parallel universes which are non-interacting among themselves.

According to the modern criteria of the scientific theories, MWI is not experimentally verificable and not falsified, and therefore is not scientific! However, any other interpretation of quantum mechanics, including the Copenhagen one, is also not scientific but philosophical and therefore the usefulness of the quantum-mechanical interpretation is determined by its pragmatism. And, although the analysis of some problems in the MWI brings to the same results as in any other interpretation, but these results are more simple logically, so they had been resulted to some physicists to be more popular in quantum mechanics (and quantum cosmology).

May be, it seems that the majority of the opponents of the MWI reject it because, for them, introducing a very large number of worlds that we do not see is an extreme violation of Ockham's principle: "Entities are not to be multiplied beyond necessity". However, in judging physical theories one could reasonably argue that one should not multiply physical laws beyond necessity either (such a verion of Ockham's Razor has been applied in the past), and in this respect the MWI is the most economical theory. Indeed, it has all the laws of the standard quantum theory, but without the collapse postulate, the most problematic of physical laws.

The reason for adopting the MWI is that it avoids the collapse of the quantum wave. And there is no ex- perimental evidence in favor of collapse and against the MWI. We need not assume that Nature plays dice. The MWI is a deterministic theory for a physical Universe and it explains why a world appears to be in-deterministic for human observers.

The MWI exhibits some kind of non-locality: "world" is a non-local concept, but it avoids action at a distance and, therefore, it is not in conflict with the relativistic quantum mechanics

The MWI is not the most accepted interpretation of quantum theory among physicists, but it is becoming increasingly popular.

The strongest proponents of the MWI can be found in the communities of quantum cosmology and quantum computing. In quantum cosmology it makes it possible to discuss the whole Universe avoiding the difficulty of the standard interpretation which requires an external observer. In quantum computing, the key issue is the parallel processing performed on the same computer; this is very similar to the basic picture of the MWI. However, the advantage of the MWI is that it allows us to view quantum mechanics as a complete and consistent physical theory which agrees with all experimental results obtained to date. And also, the elegant conception of the de-coherence, proposed in 1970 by Dieter Zeh, explains that the various branches of the single wave function, which describe these worlds, are oscillating in time with the different phases and so as if do not exist each for other [42].

As a whole, the problem of the final interpretation of quantum mechanics and of quantum theory of measurements is far from the total consensus and still remains open for both physicists and philosophers (in the science philosophy).

One can add here that the still inherent incompatibility of the postulates of quantum theory as non-local theory and relativity theory (both special and general) as local theory is the main root of the impossibility to construct the self-consistent relativistic quantum mechanics, quantum field theory and the quantum cosmology even in quasi-linear approximation.

Another long incompleteness of non-relativistic quantum mechanics (even in the Copenhagen interpretation) is connected with the problem of time as a quantum observable, which is, moreover, canonically conjugated to energy. It has been known from the beginning of twentieth of XX (see [43] and later also the discussion of Y.Aharonov and D.Bohm with Fock $[44,45]$ ) till the last years, when it has in fact been resolved practically by using the mathematical means.

\section{TIME IS REALLY A QUANTUM OBSERVABLE, CANONICALLY CONJUGATED TO ENERGY}

Introduction to the history of the problem. During almost ninety years (see, for example, [43]) it is known that 
time cannot be represented by a self-adjoint operator, with the possible exception of special abstract systems (such as an electrically charged particle in an infinite uniform electric field ${ }^{1}$ and a system with the limited from both below and above energy spectrum (to see later)). This fact results to be in contrast with the known circumstance that time, as well as space, in some cases plays the role just of a parameter, while in some other cases is a physical observable which ought to be represented by an operator. The list of papers devoted to the problem of time in quantum mechanics is extremely large (see, for instance, [46-82], and references therein). The same situation had to be faced also in quantum electrodynamics and, more in general, in relativistic quantum field theory (see, for instance, $[53,81,82]$ ).

As to quantum mechanics, the first set of known and cited articles is [54-60]. The second set of papers on time as an observable in quantum physics [61-82] appeared from the end of the eighties and chiefly in the nineties and more recently, stimulated mainly by the need of a self-consistent definition for collision duration and tunnelling time. It is noticeable that many of this second set of papers appeared however to ignore the Naimark theorem from [83], which had previously constituted an important basis for the results in refs. [54-60]. This Naimark theorem states [83] that the non-orthogonal spectral decomposition $E(\lambda)$ of a hermitian operator $H$ is of the Carleman type (which is unique for the maximal hermitian operator), i.e. it can be approximated by a succession of the self-adjoint operators, the spectral functions of which do weakly converge to the spectral function $E(\lambda)$ of the operator $H$.

Namely, by exploiting that Naimark theorem, it has been shown in [54-59] (more details having been added in $[60,65,66,78,81,82])$ that, for systems with continuous energy spectra, time can be introduced as a quantum-mechanical observable, canonically conjugate to energy. More precisely, the time operator resulted to be maximal hermitian, even if not self-adjoint. Then, in [59 $(1), 66(3), 81,82]$ it was clarified that time can be introduced also for the systems with energy discrete spectra as a quantum-mechanical observable, canonically conjugate to energy, and the time operator resulted to be quasi-self-adjoint (more precisely, it can be chosen as an almost self-adjoint operator with practically almost any degree of the accuracy).

We have also to note that there is known in the literature the so-called positive-operator-value-measure (POVM) approach, often used in the second set of papers on time

\footnotetext{
${ }^{1}$ Namely that fact that time cannot be represented by a self-adjoint operator is known to follow from the semi-boundedness of the continuous energy spectra, which are bounded from below (usually by the value zero). Only for an electrically charged particle in an infinite uniform electric field, and for other very rare special systems, the continuous energy spectrum is not bounded and extends over the whole energy axis from $-\infty$ to $\infty$.
}

in quantum physics (for instance, in [61-64, 67-77,79,80]. This approach, in general, is well-known in the various approaches to the quantum theory of measurements approximately from the sixties and had been applied in the simplest form for the time-operator problem in the case of the free motion already in [84]. Then, in [61-64, 67-77,79,80] (often with certain simpli- fications and abbreviations) it was affirmed that the generalized decomposition of unity (or POV measures) is reproduced from any self-adjoint extension of the time operator into the space of the extended Hilbert space (usually, with negative values of energy $E$ in the left semi-axis) citing the Naimark's dilation theorem from [85]. As to our approach, it is based on another Naimark's theorem (from [83]), cited above, and without any extension of the physical Hilbert space of usual wave functions (wave packets) with the subsequent return projection to the previous space of wave functions; and, moreover, it had been published in [54-57,59,60] earlier than [61-64,67-77, $79,80]$. Being based on the earlier published remarkable Naimark theorem [83], it is much more direct, simple and general, and at the same time mathematically not less rigorous than POVM approach!

From the simple analysis of the articles [57,78,81,82], based on the remarkable Naimark theorem [83], one can see that the appearance of these articles, does demonstrate that the problem of time as an observable in quantum mechanics is factually and practically resolved for the systems with continuous spectra, and the alternative approach presented in the articles [73-77,79,80], based on the another Naimark theorem [85], which does not contradict this conclusion, in fact does partially support it.

Time as a quantum observable in quantum mechanics for systems with continuous spectra. For systems with continuous energy spectra, the following simple operator, canonically conjugate to energy, can be introduced for time:

$$
\hat{t}=\left\{\begin{array}{c|l|c|c}
t & \text { in the (time) } t \text {-representation, } & (1 \mathrm{a}) \\
-i \hbar \frac{\partial}{\partial E} & \text { in the (energy) } E \text {-representation } & \text { (1b) }
\end{array}\right.
$$

which is not self-adjoint, but is hermitian, and acts on square-integrable space-time wavepackets in representation (1a), and on their Fourier-transforms in representation (1b), once the point $E=0$ is eliminated (i.e., once one deals only with moving packets, i.e., excludes any non-moving back tails, as well as, of course, the zero flux cases $)^{2}$. It has been shown already in $[54-57,59,60]$. The elimination of the point $E=0$ is not restrictive since the "rest" states with the zero velocity, the wave-packets with non-moving rear tails, and the wave-packets with zero flux are unobservable.

Operator (1b) is defined as acting on the space $P$ of the continuous, differentiable, square-integrable functions $f(E)$ that satisfy the conditions 


$$
\begin{aligned}
& \int_{0}^{\infty}|f(E)| 2 \mathrm{~d} E<\infty, \int_{0}^{\infty}|\partial f(E) / \partial E|^{2} d E<\infty \\
& \int_{0}^{\infty}|f(E)| 2 E 2 \mathrm{~d} E<\infty
\end{aligned}
$$

(the notation $<\infty$ in (2) denotes the finite value of the integrals from the left) and the condition

$$
f(0)=0
$$

which is a space $P$ dense in the Hilbert space of $L^{2}$ functions defined (only) over the semi-axis $0 \leq E<\infty$. Obviously, the operator $(1 \mathrm{a}, \mathrm{b})$ is hermitian, i.e. the relation $\left(f_{1}, \hat{t} f_{2}\right)=\left(\left(\hat{t} f_{1}\right), f_{2}\right)$ holds, only if all square-integrable functions $f(E)$ in the space on which it is defined vanish for $E=0$. And also the operator $\hat{t}^{2}$ is hermitian, i.e. the relation $\left(f_{1}, \hat{t}^{2} f_{2}\right)=\left(\left(\hat{t} f_{1}\right),\left(\left(\hat{t} f_{2}\right)\right)=\right.$ $\left(\hat{t}^{2} f_{1}, f_{2}\right)$ holds under the same conditions.

Operator $\hat{t}$ has no hermitian extension because otherwise one could find at least one function $f_{0}(E)$ which satisfies the condition $f_{0}(0) \neq 0$ but that is inconsistent with the propriety of being hermitian. So, according to [86], $\hat{t}$ is a maximal hermitian operator.

Essentially because of these reasons, earlier Pauli (see, for instance, [45]) rejected the use of a time operator: and this had the result of practically stopping studies on this subject for about forty years.

However, as far back as in [87] von Neumann had claimed that considering in quantum mechanics only self-adjoint operators could be too restrictive. To clarify this issue, let us quote an explanatory example set forth by von Neumann himself [87]: Let us consider a particle, free to move in a spatial semi-axis $(0 \leq x<\infty)$ bounded by a rigid wall located at $x=0$. Consequently, the operator for the momentum $x$-component of the particle, which reads

$$
\hat{p}_{x}=-i \hbar \frac{\partial}{\partial x},
$$

is defined as acting on the space of the continuous, differentiable, square-integrable functions $f(x)$ that satisfy the conditions

$$
\begin{aligned}
& \int_{0}^{\infty}|f(x)|^{2} \mathrm{~d} x<\infty, \int_{0}^{\infty}|\partial f(x) / \partial x|^{2} \mathrm{~d} x<\infty, \\
& \int_{0}^{\infty}|f(x)|^{2} x^{2} \mathrm{~d} x<\infty
\end{aligned}
$$

(here the notation $<\infty$ denotes the finite value of the integrals from the left) and the condition

$$
f(0)=0
$$

which is a space $Q$ dense in the Hilbert space of $L^{2}$ functions defined (only) over the spatial semi-axis $0 \leq x<\infty$. Therefore, operator $\hat{p}_{x}=-i \hbar \frac{\partial}{\partial x}$ has the same

\footnotetext{
${ }^{2}$ Such a condition is enough for operator $(1 \mathrm{a}, \mathrm{b})$ to be a "maximal hermitian" (or "maximal symmetric") operator [53-57,59,60,78,81,82], according to Akhiezer \& Glazman's terminology.
}

mathematical properties as operator $\hat{t}(1 \mathrm{a}, \mathrm{b})$ and consequently it is not a self-adjoint operator but it is only a maximal hermitian operator. Nevertheless, it is an observable with an obvious physical meaning. And the same properties has also the radial momentum operator

$$
\hat{p}_{r}=-i \hbar \frac{\partial}{\partial r}+\frac{1}{r} \quad(0<r<\infty) .
$$

By the way, one can easily demonstrate (see, for instance, [47]) that in the case of (hypotetical) quantum-mechanical systems with the continuous energy spectra bounded from below and from above $\left(E_{\min }<E<E_{\max }\right)$ the time operator $(1 \mathrm{a}, \mathrm{b})$ becomes a really self-adjoint operator and has a discrete time spestrum, with the "the time quantum" $\tau=\hbar / d$, where $d=\mid E_{\max }-$ $E_{\text {min }} \mid$.

In order to consider time as an observable in quantum mechanics and to define the observable mean times and durations, one needs to introduce not only the time operator, but also, in a self-consistent way, the measure (or weight) of averaging over time. In the simple one-dimensional (1D) and one-directional motion such measure (weight) can be obtained by the simple quantity:

$$
W(x, t) \mathrm{d} t=\frac{j(x, t) \mathrm{d} t}{\int_{-\infty}^{\infty} j(x, t) \mathrm{d} t},
$$

where the probabilistic interpretation of $j(x, t)$ (namely in time) corresponds to the flux probability density of a particle passing through point $x$ at time $t$ (more precisely, passing through $x$ during a unit time interval, centered at $t$ ), when travelling in the positive $x$-direction.. Such a measure had not been postulated, but is just a direct consequence of the well-known probabilistic ( $s p a-$ tial) interpretation of $\rho(x, t)$ and of the continuity relation

$$
\partial \rho(x, t) / \partial t+\operatorname{div} j(x, t)=0
$$

for particle motion in the field of any hamiltonian in the description of the 1D Schroedinger equation. Quantity $\rho(x, t)$ is the probability of finding a moving particle inside a unit space interval, centered at point $x$, at time $t$. The probability density $\rho(x, t)$ and the flux probability-density $j(x, t)$ are related with the wave function $\Psi$ $(x, t)$ by the usual definitions $\rho(x, t)=|\Psi(x, t)|^{2}$ and $j(x, t)=$ $\operatorname{Re}\left[\begin{array}{lll}\Psi^{*}(x, t) & (\hbar / i \mu) & \partial \Psi(x, t) / \partial x\end{array}\right]$. The measure (4) was firstly investigated in $[57,59,60,65,66]$.

When the flux density $j(x, t)$ changes its sign, the quantity $W(x, t) \mathrm{d} t$ is no longer positive definite and it acquires a physical meaning of a probability density only during those partial time-intervals in which the flux density $j(x, t)$ does keep its sign. Therefore, let us introduce the two measures, by separating the positive and the 
negative flux-direction values (i.e., flux signs):

$$
W_{ \pm}(x, t) \mathrm{d} t=\frac{j_{ \pm}(x, t) \mathrm{d} t}{\int_{-\infty}^{\infty} j_{ \pm}(x, t) \mathrm{d} t}
$$

with $j_{ \pm}(x, t)=j(x, t) \Theta( \pm j)$ where $\Theta(z)$ is the Heaviside step function. It had been made firstly in $[60,65,66]$. Actually, one can rewrite the continuity relation (5) for those time intervals, for which $j=j_{+}$or $j=j_{-}$as follows:

$$
\frac{\partial \rho_{>}(x, t)}{\partial t}=-\frac{\partial j_{+}(x, t)}{\partial x} \text { and } \frac{\partial \rho_{<}(x, t)}{\partial t}=-\frac{\partial j_{-}(x, t)}{\partial x}
$$

(the equalities (6) do formally serve also as a definitions of $\rho_{>}(x, t)$ and $\left.\rho_{<}(x, t)\right)$, respectively.

Then, one can eventually define the mean value $\langle t(x)>$ of the time $t$ at which a particle passes through position $x$ (when travelling in only one positive $x$-direction), and $<t_{ \pm}(x)>$ of the time $t$ at which a particle passes through position $x$, when travelling in the positive or negative direction, respectively :

$$
\begin{aligned}
& <t(x)>=\frac{\int_{-\infty}^{\infty} t j(x, t) d t}{\int_{-\infty}^{\infty} j(x, t) d t}= \\
& \frac{\int_{0}^{\infty} d E \frac{1}{2}\left[G^{*}(x, E) \hat{t} v G(x, E)+v G^{*}(x, E) \hat{t} G(x, E)\right]}{\int_{0}^{\infty} d E \downarrow|G(x, E)|^{2}}
\end{aligned}
$$

where $G(x, E)$ is the Fourier-transform of the moving one-dimensional (1D) wave packet

$$
\begin{aligned}
& \Psi(x, t)=\int_{0}^{\infty} G(x, E) \exp (-i E t / \hbar) \mathrm{d} E \\
& =\int_{0}^{\infty} g(E) \varphi(x, E) \exp (-i E t / \hbar) \mathrm{d} E
\end{aligned}
$$

when going on from the time representation to the energy one,

$$
<t_{ \pm}(x)>=\frac{\int_{-\infty}^{\infty} t j_{ \pm}(x, t) d t}{\int_{-\infty}^{\infty} j_{ \pm}(x, t) d t},
$$

and also the mean durations of particle 1D transmission from $x_{i}$ to $x_{f}>x_{i}$ and 1D particle reflection from the region $\left(x_{i}, \infty\right)$ into $x_{f} \leq x_{i}$ :

$$
\begin{gathered}
<\tau_{T}\left(x_{i}, x_{f}\right)>=<t_{+}\left(x_{f}\right)>-<t_{+}\left(x_{i}\right)>\text { and } \\
<\tau_{R}\left(x_{i}, x_{f}\right)>=<t_{-}\left(x_{f}\right)>-<t_{+}\left(x_{i}\right)>,(7 \mathrm{c})
\end{gathered}
$$

respectively. Of course, it is possible to pass in Eq.7b also to integrals $\int_{0}^{\infty} d E \ldots$, similarly to (7a) and (8) by using the unique Fourier (Laplace) - transformations and the energy expansion of $j_{ \pm}(x, t)=j(x, t) \Theta( \pm j)$, but it is evident that they result to be rather bulky. The generalization for the three-dimensional motions is given in [82].

Now, one can see that two canonically conjugate operators, the time operator (1) and the energy operator

$$
\hat{E}=\left\{\begin{array}{cl}
E & \text { in the energy }(E-) \text { representation, } \\
-i \hbar \frac{\partial}{\partial t} & \text { in the time }(t-) \text { representation }
\end{array}\right.
$$

satisfy the typical commutation relation

$$
[\hat{E}, \hat{t}]=i \hbar \text {. }
$$

Although up to now according to the Stone and von Neumann theorem [88] the relation (10) has been interpreted as holding only for the pair of the self-adjoint canonically conjugate operators, in both representations, and it was not directly generalized for maximal hermitian operators, the difficulty of such direct generalization has in fact been by-passed by introducing $\hat{t}$ with the help of the single-valued Fourier(Laplace)-transformation from the $t$-axis $(-\infty<t<\infty)$ to the $E$-semi-axis $(0<E<\infty)$ and by utilizing the peculiar mathematical properties of maximal hermitian operators.

Actually, from Eq.10 the uncertainty relation

$$
\Delta E \Delta t \geq \hbar / 2
$$

(where the standard deviations are $\Delta a=\sqrt{ } \mathrm{D} a$, quantity $\mathrm{D} a$ being the variance $\mathrm{D} a=\left\langle a^{2}\right\rangle-\langle a\rangle^{2}$; and $a=E, t$, while $\langle\ldots>$ denotes an average over $t$ by the measures $W(x, t) \mathrm{d} t$ or $W_{ \pm}(x, t) \mathrm{d} t$ in the $t$-representation or an average over $E$ similar to the right-hand-part of (7a) and (8) in the $E$-representation) was derived by the simple generalizing of the similar procedures which are standard in the case of self-adjoint canonically conjugate quantities. Moreover, relation (10) satisfies the Dirac "correspondence principle", since the classical Poisson brackets $\left\{q_{0}, p_{0}\right\}$, with $q_{0}=t$ and $p_{0}=-E$, are equal to unity [89]. In [57] (see also [59]) it was also shown that the differences between the mean times at which a wave-packet passes through a pair of points obey the Ehrenfest correspondence principle; in other words, in $[57,59]$ the Ehrenfest theorem was suitably generalized.

After what precedes, one can state that, for systems with continuous energy spectra, the mathematical properties of the maximal hermitian operators (described, in particular, in [57,59]), like $\hat{t}$ in Eq.1, are sufficient for considering them as quantum observables: Namely, the uniqueness of the "spectral decomposition" (also called spectral function) for operators $\hat{t}$, as well as for $\hat{t}^{n}$ $(n>1)$ guarantees (although such an expansion is not 
orthogonal) the equivalence of the mean values of any analytic functions of time, evaluated either in the $t$ - or in the $E$-representations. In other words, the existence of this expansion is equivalent to a completeness relation for the (formal) eigen functions of $\hat{t}^{n}(n \geq 1)$, corresponding with any accuracy to real eigen values of the continuous spectrum; such eigen functions belonging to the space of the square integrable functions of the energy $E$ with the boundary conditions like (2)-(3) (see details in $[81,82])$.

From this point of view, there is no practical difference between self-adjoint and maximal hermitian operators for systems with continuous energy spectra.

Time as a quantum observable in quantum mechanics for systems with discrete spectra. For systems with discrete energy spectra it is natural (following [59,81,82]) to introduce wave packets of the form

$$
\psi(x, t)=\sum_{n=0} g_{n} \varphi_{n}(x) \exp \left[-i\left(\varepsilon_{n}-\varepsilon_{0}\right) t / \hbar\right]
$$

(where $\varphi_{n}(x)$ are orthogonal and normalized wave functions of system bound states which satisfy equation $\hat{H} \varphi_{n}(x)=\varepsilon_{n} \varphi_{n}(x), \hat{H}$ being the system Hamiltonian; $\sum_{n=0}\left|g_{n}\right|^{2}=1$; here we factually omitted a non-significant phase factor $\exp \left(-i \varepsilon_{0} t / \hbar\right)$ as being general for all terms of the sum $\sum_{n=0}$ ) for describing the evolution of systems in the regions of the purely discrete spectrum. Without limiting the generality, we choose moment $t=0$ as an initial time instant.

Firstly, we shall consider those systems, whose energy levels are spaced with distances for which the maximal common divisor is factually existing. Examples of such systems are harmonic oscillator, particle in a rigid box and spherical spinning top. For these systems the wave packet (12) is a periodic function of time with the period (Poincaré cycle time) $T=2 \pi \hbar / D, D$ being the maximal common divisor of distances between system energy level.

In the $t$-representation the relevant energy operator $\hat{H}$ is a self-adjoint operator acting in the space of periodical functions whereas the function $t \psi(t)$ does not belong to the same space. In the space of periodical functions the time operator $\hat{t}$, even in the eigen representation, has to be also a periodical function of time $t$. This situation is quite similar to the case of azimuth momentum $\varphi$, canonically conjugated to angular momentum $\hat{L}_{z}$ (see, for instance, $[90,91])$. Utilizing the example and result from [92], let us choose, instead of $t$, a periodical function

$$
\hat{t}=t-T \sum_{n=o}^{\infty} \Theta(t-[2 n+1] T / 2)
$$

$$
+T \sum_{n=o}^{\infty} \Theta(-t-[2 n+1] T / 2)
$$

which is the so-called saw-function of $t$ (see Figure 1).

This choice is convenient because the periodical function of time operator (13) is linear function (one- directional) within each Poincaré interval, i.e. time conserves its flowing and its usual meaning of an order parameter for the system evolution.

The commutation relation of the self-adjoint energy and time operators acquires in this case (discrete energies and periodical functions) the form:

$$
[\hat{E}, \hat{t}]=i \hbar\left\{1-T \sum_{n=0}^{\infty} \delta(t-[2 n+1] T)\right\} .
$$

Let us recall (see, e.g. [92]) that a generalized form of uncertainty relation holds

$$
(\Delta A)^{2} \cdot(\Delta B)^{2} \geq \hbar^{2}[<N>]^{2}
$$

for two self-adjoint operators $\hat{A}$ and $\hat{B}$, canonically conjugate each to other by the commutator

$$
[\hat{A}, \hat{B}]=i \hbar \hat{N} \text {, }
$$

$\hat{N}$ being a third self-adjoint operator. One can easily obtain

$$
(\Delta E)^{2} \cdot(\Delta t)^{2} \geq \hbar^{2}\left[1-\frac{\mathrm{T}|\psi(\mathrm{T} / 2+\gamma)|^{2}}{\int_{-\mathrm{T} / 2}^{+\mathrm{T} / 2}|\psi(t)|^{2} d t},\right.
$$

where the parameter $\gamma$ (with an arbitrary value between $-T / 2$ and $+T / 2$ ) is introduced for the univocality of calculating the integral on right part of (17) over $d t$ in the limits from $-T / 2$ to $+T / 2$, just similarly to the procedure introduced in [90] (see also [92]).

From (17) it follows that when $\Delta E \rightarrow 0$ (i.e. when $\left|g_{n}\right| \rightarrow \delta_{n n}$, the right part of (17) tends to zero since $|\psi(t)|^{2}$ tends to a constant. In this case the distribution of time instants of wavepacket passing through point $x$ in the

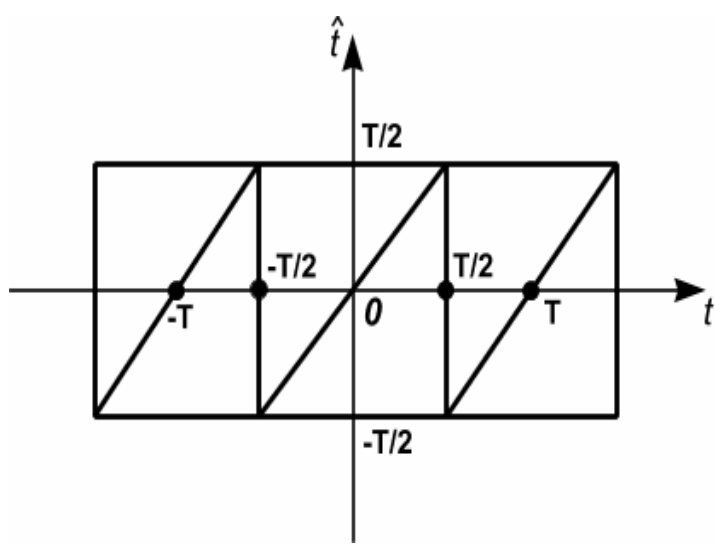

Figure 1. The periodical saw-tooth function for time operator in the case of (13). 
limits of one Poincaré cycle becomes uniform. When

$\Delta E>>D$ and $|\psi(T+\gamma)|^{2}<<T^{-1} \cdot \int_{-T / 2}^{\mathrm{T} / 2}|\psi(t)|^{2} \mathrm{~d} t$,

the periodicity condition may be inessential for $\Delta t<<T$, i.e. (17) passes to uncertainty relation (11), which is just the same one as for systems with continuous spectra.

One can also obtain the expression for the time operator (13) in energy representation $[59,82]$.

In general cases, for excited states of nuclei, atoms and molecules, level distances in discrete spectra have not strictly defined the maximal common divisor and hence they have not the strictly defined time of the Poincaré cycle. And also there is no strictly defined passage from the discrete part of the spectrum to the continuous part. Nevertheless, even for those systems one can introduce an approximate description (and with any desired degree of the accuracy within the chosen maximal limit of the level width, let us say, $\gamma_{\text {lim }}$ ) by quasi-cycles with quasi-periodical evolution and for sufficiently long intervals of time the motion inside such systems (however, less than $\hbar / \gamma_{\text {lim }}$ ) one can consider as $a$ periodical motion also with any desired accuracy. For them one can choose (define) a time of the Poincare' cycle with any desired accuracy, including in one cycle as many quasi-cycles as it is necessary for demanded accuracy. Then, with the same accuracy the quasiself-adjoint time operator (13) can be introduced and all time characteristics can be defined.

In the degenerate case when at the state (12) the sum $\sum_{n=0}^{\infty}$ contains only one term $\left(g_{n} \rightarrow \delta_{n n}\right)$, the evolution is absent and the time of the Poincare' cycle is equal formally to infinity.

For systems with continuous and discrete regions of the energy spectrum, one uses both forms: (1) for the continuous energy spectrum and (13) for the discrete energy spectrum. As a concluding remark, it is possible to state that the mathematical properties of $\hat{t}_{\text {and }} \hat{t}^{n}$ $(n>1)$ are quite enough for considering time as a quantum-mechanical observable (like for energy, momentum, spatial coordinates,...) without having to introduce any new physical postulates.

Time analysis of quantum processes, based on time operator. Let us limit ourselves here by only some known results and perspectives:

1) Now there are certain foundations to accept $[81,82]$ that energy-time uncertainty relations (11) with (17) can help to attenuate endless debates on their interpretation, originated in $[44,45]$.

2) Time analysis of the motion of the non-relativistic particles and photons revealed not only the similarity in the motion of particles and photons [93-95] (see also [66(2 and 3),78,81,82]) but did also brought to the introduction of the maximal hermitian time operator for quantum electrodynamics (at least, for the 1D photon motion $[78,81,82])$.

3) There are already known two measures of averaging on time in quantum mechanics. Earlier it was reported on the first measure which is related with the particle passing through space point or interval (volume). The second measure is related with the particle accumulation or dwelling (or sojourning) inside the limited space interval (volume) during passing through it (it is described, in particular in reviews [78,81,82]).

4) Actually, the time operator (1) has been rather fruitfully used in the case of the tunnelling times and, generally, in the time analysis of tunnelling processes. It had established that practically all earlier known particular tunnelling times appear to be the special cases of the mean tunnelling time or of the square root of the variance in the tunnelling-time distribution (or pass into them under some boundary conditions), defined within the general quantum-mechanical approach. It had been carried out in some reviews (in particular, in [78,81,82], see also [96]). Then, a lot of other interesting results concerning time behaviour of tunnelling particles and photons inside a barrier had been revealed, including the experimental revealing of the superluminal group velocities of tunnelling photons [97-99].

It is meaningful to stress also that, although any direct classical limit for particle tunnelling through potential barrier with sub-barrier energies is really absent, there is the direct classical limit for the wave-packet tunnelling. Let us recall real evanescent and anti-evanescent waves, well-known in classical optics and in classical acoustics (as it was, for instance, mentioned in [78 (conclusions IV and V)] and, in a more detailed manner, in [82 (conclusion 4)]).

5) An actual perspective for the nearest future is opened for generalizing the time analysis of quantum processes for more complicated particle and photon motions (for instance, such as along helixes and motions through two-dimensional and three-dimensional (including non-spherical) potential barriers etc).

6) Similar derivations and conclusions with quite evident generalization can be carried out for time operator in relativistic quantum mechanics (the Klein-Gordon case and the Dirac case). It is rather perspective (but, of course, not always simple) to develop the analysis of four-position operators for other relativistic cases, especially to analyze the localization problems. A review of the preliminary results on this topic is already appeared [100].

\section{MORE DETAILED COMMENTS ON THE COMPLEX OF PROBLEMS CONNECTED WITH THE ORIGIN OF THE BIOLOGIC LIFE}

Now let us analyze, in a condensed way, one of the great 
natural problems marked in [13] - the problem of the reductionism of biology to physics (including, first of all, the problem of the physical and chemical explanation of the origin of the biologic life).

Explanation of the origin of the biologic life in terms of physics and, in general, of natural sciences (chemistry etc., including also mathematics) $\leftrightarrow$ there is a problem of the origin of the genetics, genetic code (or at least a small set of several codes) which is unique for all the terrestrial biosphere, and the defense mechanisms for the defense of the organism development during cell reproduction,...

$\leftrightarrow$ there is an inevitable choice (dilemma): either a natural event (or process) like a certain jump which is similar to some kind of phase transition (or like to synergetic process, or even like the irrational many-world interpretation), or a supreme intelligent design of a super-human creative basis (or a Creator).

Any attempt of the natural origin is failed. And not only because the self-origin of only one self-reproducing cell has not a scientifically reliable explanation in the limits of modern physics (the probability of the chance formation of the protein configuration, containing still 500 nucleotides, is extremely small, i.e. near $1 / 10^{950}$, and for the cell formation it is necessary at least 250 different proteins). There are no scientific explanations yet even for the following facts and no answers for the following problems:

How a numerous quantity of the chemical reactions could take place in a very limited space volume for create one protein molecule?

How there were created the conditions, which were necessary for uniting some components and at the same time were unfavorable for uniting other components, and how then the successive creation of a protein (or RNA or DNA) molecule can happen?

If even a principal possibility of the formation of the simplest protein components (DNA) had been shown in the known Oparin, Miller (etc) experiments under the special laboratory conditions, all the same it is very remote from the conditions of the primordial earth or of the unstable cosmos. So, no terrestrial or cosmic origin of cells (moreover, with the genetic structure) are impossible!

And how one can explain that

a) The genetic information in the DNA can be read only by the specific ferments, for the creation of which the special information is also coded in the DNA.

$b$ ) The biochemical process of the protein synthesis is the most complicated process between all known biochemical processes in the cell, and also some protein is already necessary for the protein production. Then, the genetic code is beforehand required for the information transfer from the DNA to the protein, and such code is almost universal for the whole terrestrial biosphere.

c) And finally, the genetic code has the vitally neces- sary control system, which is, in its turn, is coded in the DNA.

It is impossible to explain all these facts in the natural way.

d) And how one (or almost one) main genetic code for the whole terrestrial biosphere had been originated?

Nobody could elaborate somehow working model of the origin of even one self-reproducing cell yet.

The first main part of this problem of the origin consists in the absence of the answer to the following question: how had been originated the conditions, which are vitally necessary for living systems now, during that time when the life had been absent but which are created by only living systems! So, it is absolutely unclear: what had been earlier - habitat with is necessary for the life, or the living organisms in the medium which had not supported the life.

The second main part of this problem consists in the mystery of the origin of the enormous quantity of the coded genetic information.

Finally, there is no doubt that the whole terrestrial biosphere is a wonderfully balanced eco-system of the irreducible complexity and integrity. The interaction of all its components (flora, fauna, micro-organisms and habitat) is such that the disappearance of even if one of them will bring to the disappearance of the whole biosphere.

So, it is not surprising that during the last ten (or somewhat more) years the number of scientific papers dedicated to the critics of the natural evolutional biologic and pre-biologic theories has become to increase [101-104].

There some, may be, naturalists who do still hope that certain synergetic processes can initiate the self-organization of the non-living matter into the living organisms. But now it is known (see, for instance, [105]) that all concrete macroscopic systems with known history of their origin, which are more highly ordered than their environment, were created not by rare occasional fluctuations, but under the direct influence of external forces or as a result of bifurcations caused by some non-linearity and external forces in the open systems. Moreover, I.Prigogine denied that revealed by him processes of local decreasing of entropy can explain the origin of the alive from the non-alive [106]: "The point is that in a non-isolated system there exists a possibility for formation of ordered, low-entropy structures at sufficiently low temperatures. This ordering principle is responsible for the appearance of ordered structures such as crystals as well as for the phenomena of phase transitions. Unfortunately this principle cannot explain the formation of biological structures."

Returning to the direct analysis of the problem of the reductionism of biology to physics in the narrow sense ("if the biology (at least molecular biology and genetics) can be totally explained in terms of physics (and chem- 
istry)"), I can recommend to pay a particular attention to the discussion on the special problem of the principal possibility of the explanation of the cell self-reproduction in terms of quantum mechanics, initiated by E.Wigner [107], then continued by M.Eigen [18] and afterwards analyzed by M.V.Vol'kenstein [109]. Firstly, E.Wigner had simply demonstrated that really in the stochastic quantum-mechanical description the process of the cell self-reproduction cannot be explained by quantum mechanics. Then M.Eigen had shown that the possibility of the cell self-reproduction can be explained by quantum mechanics if and only if the evolution matrix (the $S$-matrix of the process) is specially instructed for this aim. Further M.V.Vol'kenstein in his analytic review [109] had expressed his expectation that M.Eigen in his future study of the pre-biologic evolution can find the possibility of such special instruction. But up to now nobody had revealed such possibility! As to me, I can see only a certain similarity (of course, partial) between two kinds of processes (with are more intellectual than naturalistic, by the way): between the process of the human writing of certain scientific files in modern computer devices and the process of the supreme-Intelligence-design writing of certain genetic programs (including the genetic program of the cell reproduction) in cells of alive organisms.

\section{MORE DETAILED COMMENTS ON THE COMPLEX OF PROBLEMS CONNECTED WITH THE UNIVERSE ORIGIN}

1) Earlier, after Enlightenment till approximately 1920 , scientists in the natural sciences did usually consider the Universe as eternally existing and eternally moving.

Now the most convincing arguments against the model of the eternally existing Universe are:

a) the second law of thermodynamics which does inevitably bring to the heat death of the Universe,

b) the observed cosmic microwave background .

The most surprising conclusion of the revealed nonstationary state of the Universe is the existence of the "beginning", under which the majority of physicists understand the beginning of the Universe expansion.

The cosmologic problem as the problem of the origin and evolution of the Universe has initiated to be analyzed by A.Einstein (after 1917) and now it is connected with papers of many other physicists. The first several authors had been G.Lemaître (who proposed what became known as the Big Bang theory of the origin of the Universe, although he called it his "hypothesis of the primeval atom"), A.Friedman and G.Gamow.

And what namely had been in the "beginning"? Gamow had assumed in 1921 that the expansion had initiated from the super-condensed hot state as a result of the
Big Bang, to which he and others had ascribed the time moment $t=0$, i.e. the beginning of the Universe history. The initial state in this model is postulated. However, the nature of the initial super-condensed hot Universe state is not known. Such initial point (or super-small region), in which the temperature, pressure, energy density etc had reached the anomalous huge (almost infinite) values, can be considered as a particular point, where The "physical" processes cannot be described by physical equations and in fact are excluded from the model analysis.

Strictly speaking, namely in the region of this point (from $t=0$ till $\sim t_{0}=10^{-44} \mathrm{sec}$, where $t_{0}$ is the Planck time) is arising the general problem of the world origin and also the choice dilemma: the beginning of the Universe formation from vacuum ("nothing") is either a result of the irrational randomness after passing from other space-time dimensions or from other universe, caused by some unknown process, or a result of the creation of the expanding Universe (together with the laws of its functioning) by the supreme intelligent design from nigilo.

The framework for the standard cosmologic model relies on Albert Einstein's general relativity and on simplifying assumptions (such as homogeneity and isotropy of space). There are even non-standard alternative models. Now there are many supporters of Big Bang models. The number of papers and books on standard and non-standard versions of the cosmologic Big Bang models is too enormous for citing in this not very large paper (it is possible to indicate, only for instance, [110-113] for initial reading in cosmology of the Universe and in the different quasi-classical and quantum approaches in cosmology for description of the creation and the initial expansion of the Universe). However, there is no well-supported model describing the action prior to $10^{-15}$ seconds or so. Apparently a new unified theory of quantum gravitation is needed to break this barrier. Understanding this earliest of eras in the history of the Universe is currently one of the greatest unsolved problems in physics.

Moreover, it is worth to underline that many physicists consider that the second law of thermodynamics is universal for all closed systems, including also our Universe as a whole (which is closed in naturalistic oneworld view). Therefore the heat death is inevitable (see, for instance, [13] and especially [114]). Finally, to-day a lot of attention of researchers is dedicated to the problems of the hypothesis of dark mass and of dark energy.

2) From 1973 (and particularly after eighties) the term "anthropic principle", introduced by B.Carter, has become to acquire in the science and out of the science a certain popularity $[115,116]$. Carter and other authors had been noted that physical constants must have values in the very narrow interval in order the existence of the biologic life can become possible, and that the measured 
values of these constants are really found in this interval. In other words, the Universe seems to be exactly such as it is necessary for the origin of the life. If physical constants would be even slightly other, then the life could be impossible.

After meeting such testimonies, a number of scientists had formulated several interpretations of anthropic principle each of which brings the researchers to the worldview choice in its peculiar way. We shall consider here two of them.

According to the weak anthropic principle (WAP), the observed values of physical and cosmological constants caused by the necessary demand that the regions, where the organic life would be developed, ought to be possible. And in the context of WAP there is the possibility of choice between two alternatives:

1) Either someone does irrationally believe that there are possible an infinity of universes, in the past, in the present and in the future, and we exist and are sure in the existence of our Universe namely because the unique combination of its parameters and properties could permit our origin and existence.

2) $\mathrm{Or}$ someone does (also irrationally) believe that our unique Universe is created by Intelligent Design of a Creator (or God) and the human being is also created by Creator in order to govern the Universe.

According to the strong anthropic principle (SAP), the Universe has to have such properties which permit earlier or later the development of life. This form of the anthropic principle does not only state that the universe properties are limited by the narrow set of values, compatible with the development of the human life, but does also state that this limitation is necessary for such purpose. So, one can interpret such tuning of the universe parameters as the testimony of the supreme intelligent design of a certain creative basis. There is also a rather unexpected interpretation of SAP, connected with the eastern philosophy, but it is not widely known.

\section{CONCLUSIONS}

It is proposed firstly a novel division of the different classes of natural sciences (and in some degree of all sciences) with different objects and paradigms: a) the entirely natural sciences, b) the natural sciences with the essential role of the human factor, or with the human intelligent design, in their objects and c) the sciences, implicated in the origin and the subsequent history of such natural "meta-systems" as the whole Universe and the whole (terrestrial) biosphere.

Several reasons caused here to formulate a new retrospective view of the science history (especially in the field of natural sciences) in XX-XXI: Firstly, under the influence of scientific and technological progress it has been intensified such direction of the science philosophy as the scientific realism (i.e. the correspondence of the science to the reality), which has in turn changed three forms: from the naïve realism to the usual realism and then to the critical scientific realism (the last one had been developed under the strong influence of sharp discussions in quantum mechanics). Secondly, some big problems of physics and natural sciences a) sharp problems and paradoxes revealed in the development of quantum mechanics and quantum theory of measurements, b) a huge complex of the problems connected with the Universe origin and the expansion after the Big Bang, c) the open problem of the origin of the biological life) have been gradually concentrated the attention of the researchers, if not scientifically but at least philosophically, to those problems as to the grand or great problems. And thirdly, the analysis of mathematics in different sciences, beginning from physics, shows that mathematics did now become the branch of the natural sciences (namely of theoretical physics) and in fact generated the final solution of the old problem of time as a quantum observable.

The interpretation questions in the considered here grand and great problems of natural sciences are practically inevitably connected with the world-views of the researchers. Therefore, it is quite clear that the strong divergences in the various interpretations and even paradigms of various researchers, especially relating to these grand and great open problems, can be caused by the incompatibility of their world-views.

Such phenomena, as 1) the enhancement of the philosophy of the critical scientific realism, 2) the problems, the paradoxes and the variety of interpretations in quantum theory, 3) the open problems of origin of the Universe and 4) the unresolved problem of the origin of the biosphere, 5) the clear extension of the role of mathematics in physics and other sciences, 6) the competition of various interpretations and even of the worldviews of researchers in the study of the great and grand problems, are the important peculiarities of the history of natural sciences in XX-XXI, which in many respects define and pre-determine the further science history.

\section{REFERENCES}

[1] Boyd, R. (1983) On the current status of the issue of scientific realism. Erkenntnis, 45-50.

[2] Bird, A. (1998) Philosophy of science, UCL Press, Montreal, McGill-Queen's University Press, London, 124.

[3] Niiniluotto, I. (2002) Critical scientific realism. Oxford University. Press.

[4] Quine, W.V.O. (1968) Ontological Relativity. Journal of Philosophy, LXV, 7, 185-212.

[5] Polkinghorne, J. (1991) Reason, and Reality, The Rela- 
tionship Between Science and Theology, Trinity Press Internat, Philadelphia.

[6] McGrath, A.I. (2004) The science of God, T\&T Clark International, London, 139-152.

[7] Kuhn, T.S. (1962; 2nd edition, 1970; 3rd edition, 1996) The Structure of Scientific Revolutions, University of Chicago Press, Chicago, IL.

[8] Einstein, A. (1944) Remarks on Russel's theory of knowledge, Philosophy of Bertrand Russel, Schlipp, A., Muschalek, H., Ed. (1950, 2nd ed.) Dio e gli scienziati [Translation in Italian from German (Original title: Gottbekenntnisse moderner Naturforscher) by Valeria Cremona ], Alba: Paoline, , 421, 30-31.

[9] von Wolfgang Sartorius von Waltershausen (1856, repr. 1965) Gauss zum Gedächtniss, Sändig Reprint Verlag H. R. Wohlwend.

http://www.amazon.de/Gauss-Gedächtnis-Wolfgang-Sart orius-Waltershausen/dp/3253017028

http://en.wikipedia.org/wiki/Mathematics. von Wolfgang Sartorius von Waltershausen (Autor)

[10] Popper, K.R. (1995) In Search of a Better World: Lectures and Essays "On knowledge", from Thirty Years. Routledge.

[11] Bogolyubov, N.N., Medvedev, B.V. and Polivanov, M.K. Ed., Dispersion relations, transactions Problems of modern Physics, N2 [in Russian: Боголюбов Н.Н., Медведев Б.В., and Поливанов М.К. (редакторы), (1957) Дисперсионные соотношения, статьи в сб. Проблемы современной физики, 2 ]; Arnold V.I., What is mathematics?, Moscow, MCNMO-press $<3$ [in Russian: Арнольд В.И., Что такое математика? М., изд-во МЦНМО, 2008, стр.3].

[12] Galilei, G. (1623) II Saggiatore (in Italian) (Rome)

[13] Ginzburg, V.L. (1999) What problems of physics and astrophysics seem now to be especially important and interesting (30 years later, already on the verge of XXI century), Physics - Uspekhi, 42, 353-272; (2002) On some advances in physics and astronomy over the past 3 years, 45, 205-211.

[14] Einstein, A., Podolsky, B. and Rosen, N. (1935) Can Quantum-Mechanical Description of Physical Reality be Considered Complete? Physical Review, 47, 777-780.

[15] Bohm, D. (1952) A Suggested Interpretation of the Quantum Theory in Terms of "Hidden Variables" I, Physical Review, 85, 66-179; (1952) A Suggested Interpretation of the Quantum Theory in Terms of "Hidden Variables" II, Physical Review, 85, 80-193; Bohm,D., Aharonov, Y. (1957) Discussion of Experimental Proof for the Paradox of Einstein, Rosen, and Podolsky, Physical Review, 108, 1070-1076; Aharonov, Y. and Bohm D., (1959) Significance of electromagnetic potentials in the quantum theory. Physical Review, 115, 485-491; Bohm, D. and Aharonov, Y. (1960) Further discussion of possible experimental tests for the paradox of einstein, Podolsky and Rosen, N.C. 17, 964; Aharonov, Y. and Bohm D. (1962) Remarks on the possibility of quantum electrodynamics without potentials, Physical Review, 125, 192; Aharonov, Y. and Bohm D. (1963) Further discussion of the role of electromagnetic potentials in the quantum theory. Physical Review, 130, 1625.

[16] Bell, J.S. (1964) On the Einstein-Podolsky-Rosen paradox. Physics, 195-200 [Bell, J.S. (1965)On the Ein-
stein-Poldolsky-Rosen paradox, Physics 1, 195-200] ,Bell, J.S, (1987) Speakable and unspeakable in quantum mechanics. Cambridge University Press.

[17] Pais, A. (1979) Einstein and the quantum theory. Reviews of Modern Physics, 51, 863-914.

[18] Popper, K. (1982) A critical note on the greatest days of quantum theory. Foundations of Physics, 12, 971-976.

[19] Holland, P.R. (1993) The quantum theory of motion: an account of the de broglie-bohm causal interpretation of quantum mechanics. Cambridge University Press, Cambridge.

[20] Mermin, N.D. (1993) Hidden variables and the two theorems of john bell. Reviews of Modern Physics, 65, 803-815.

[21] Paty, M. (1995) The nature of Einstein's objections to the copenhagen interpretation of quantum mechanics. Found. Phys., 25, 183-204.

[22] Dürr, D., Goldstein, S. and Zanghì, N. (1997) Bohmian Mechanics and the Meaning of the Wave Function, in Cohen, R.S., Horne, M., and Stachel, J., Eds., Experimental Metaphysics - Quantum Mechanical Studies for Abner Shimony, 1; Boston Studies in the Philosophy of Science, 193, Boston: Kluwer Academic Publishers; Dürr, D. (2001) Bohmsche mechanik als grundlage der quantenmechanik. Springer-Verlag. Berlin

[23] Hardy, L. (1993) Non-locality for 2 particles without inequalities for almost all entangled states. Physical Review Letters, 71, 1665-1668; Sakurai, J.J. (1994) Modern Quantum Mechanics. Addison-Wesley, USA, (see 174187, 223-232).

[24] Vaidman, L. (1994) Teleportation of quantum states. Physical Review, A, 49, 1473-1476.

[25] Brassard, G., Braunstein, S., and Cleve, R. (1998) Teleportation as a quantum computation. Physical D, 120, 43-47.

[26] Bouwmeester, D., Pan, J.-W., Mattle, K., Eibl, M., Weinfurter, H., and Zeilinger, A. (1997) Experimental quantum teleportation. Nature, 390(6660), 575-579.

[27] Boschi, D., Branca, S., De Martini, F., Hardy, L. and Popescu, S. (1998) Experimental realization of teleporting an unknown pure quantum state via dual classical and Einstein-Podolsky-Rosen channels, Physical Review Letter, 80(6), 1121-1125.

[28] Kilin, S.Y. (2001) Quanta and information, Progress in optics, 42, 1-90.

[29] Riebe, M., Häffner, H., Roos, C. F., Hänsel, W., Ruth, M., Benhelm, J., Lancaster, G. T., Körber, T. W., Becher, C., Schmidt-Kaler, F., James, D.F.V., and Blatt, R., (2004) Deterministic quantum teleportation with atoms. Nature, 429, 734-737.

[30] Ursin, R., et al. (2004) Quantum teleportation link across the danube. Nature, $\mathbf{4 3 0}, 849$.

[31] Olmschenk, S., et al. (2009) Quantum teleportation between distant matter qubits. Science, 323, 486.

[32] Everett, H. (1957) Relative State Formulation of quantum mechanics. Review of Modern Physics, 29, 454-462.

[33] De Witt, B.S.M. (1970) Quantum mechanics and reality. Physics Today, 23, 30-35.

[34] Everett, H. (1973) The theory of the universal wave function. De Witt, B. and Graham, N. Eds., The Many-Worlds Interpretation of Quantum Mechanics, Princeton NJ: Princeton University Press. 
[35] Deutsch, D. (1986) Three experimental implications of the Everett interpretation, in Penrose, R. and Isham, C.J., Ed., Quantum Concepts of Space and Time, The Clarendon Press, Oxford, 204-214.

[36] Tipler, D. (1986) The many-worlds interpretation of quantum mechanics in quantum cosmology. in Penrose, R. and Isham, C.J. Eds., Quantum Concepts of Space and Time, The Clarendon Press, Oxford, 204-214.

[37] Albert, D. and Loewer, B. (1988) Interpreting the many worlds interpretation. Synthese, 77, 195-213.

[38] Barvinsky, A.O. and Kamenshchik, A.Y. (1995) Preferred basis in quantum theory and the problem of classicalization of the quantum universe. Physical Review D, 52, 743-757.

[39] Deutsch, D. (1996) The fabric of reality, The Penguin Press, New York.

[40] Lockwood, M., Brown, H.R., Butterfield, J., Deutsch, D., Loewer, B., Papineau, D. and Saunders, S. (1996) Symposium: The "many minds" interpretation of quantum theory. British Journal for the Philosophy of Science, 47, 159-248.

[41] Barrett, J.A. (1999) The quantum mechanics of minds and worlds. Oxford: University Press.

[42] Zeh, H.D. (1970) On the interpretation of measurement in quantum theory. Foundations of Physics, 1, 69-76; Zeh, H.D. (1973) Toward a quantum theory of observation. Foundations of Physics, 3, 109-116.

[43] Pauli, W. (1926) in: Handbuch der Physik, 5(1), 60, ed. by Fluegge, S. (Berlin),; see also: Pauli, W. General principles of quantum theory. (Springer; Berlin, 1980).

[44] Aharonov, Y. and Bohm, D. (1961) Time in the quantum theory and the uncertainty relation for time and energy, Physical Review, 122, 1649-1658; Aharonov, Y. and Bohm, D. (1964) Answer to fock concerning the time energy indeterminacy relation. Physical Review B, 134, 1417-1418.

[45] Krylov, N.S. and Fock, V.A. (1947) On two main interpretations of energy-time uncertainty. Sov. J. Zhetf, 17, 93-99; Fock, V.A. (1962) On the energy-time uncertainty and on an attempt to refute it. Sov. J. Zhetf, 42, $1135-1140$.

[46] Paul, H. (1962) Über quantenmechanische Zeitoperatoren. Annalen der Physik, 9, 252-261.

[47] Engelman, F. and Fick, E. (1963) Quantentheorie der Zeitmessung. Zeitschrift für Physikalische A, 175, 271282; (1964) Quantentheorie der Zeitmessung-II. Zeit-s chrift für Physikalische A, 178, 551-562.

[48] Lippmann, B.A., (1966) Operator for time delay induced by scattering. Physical Review, 151, 1023-1024.

[49] Razavy, M. (1969) Quantum-mechanical conjugate to the Hamiltonian operator. Nuovo Cimento, B, 63, 271-308.

[50] Gien, T.T., (1969) On the operators for time of motion and delay time induced by scattering. Can. Journal of Physics, 47, 278-289; (1970) Delay time and phase of the initial state. Canadian Journal of Physics, 48, 639-652.

[51] Allcock, G.R. (1969) The time of arrival in quantum mechanics. Annals of Physics (N.Y.), 53, 253-285.

[52] Rosenbaum, D.M. (1969) Super hilbert space and the quantum-mechanical time operators. Journal of Mathematical Physics, 10, 1127-1144.

[53] Olkhovsky, V.S. and Recami, E. (1968) Space-time shifts and cross sections in collisions between relativistic wane packets. Nuovo Cimento, A53, 610-624; (1969) About collision-process lifetimes and causality. Nuovo Cimento $A, 63,814-826$; (1970) About a space-time operator in collision descriptions. Nuovo Cimento, 4, 1165-1173.

[54] Olkhovsky, V.S. (1973) On the problem of time operator and collision duration, Ukrainskiy Fiz. Zhurnal [in Ukrainian and Russian], 18, 1910-1913; Olkhovsky, V.S., Recami, E. and Gerasimchuk, A.I. (1974) Time operator in quantum mechanics - non-relativistic case. Nuovo Cimento A, 22, 263-278.

[55] Recami, E. (1977) A time operator and the time-energy uncertainty relation. in The Uncertainty Principle and Foundation of Quantum Mechanics (J.Wiley; London), 21-28; "An operator for observable time", in Proceeding of the XIII Winter School in Theor. Physics, (Wroclaw; 1976), 2, 251-256.

[56] Holevo, A.S. (1978) Estimation of shift parameters of a quantum state. Re Math. Phys., 13, 379-399; Holevo, A.S. (1982) Probabilistic and statistical aspects of quantum theory, Amsterdam.

[57] Olkhovsky, V.S. (1984) To the investigation of nuclear reactions and decays by analysis of their durations. Soviet Journal Nuclear Physics, 15, 130-148.

[58] Jaworski, W. and Wardlaw, D.M. (1988) Time delay in tunneling: Transmission and reflection time delays. Physical Review A, 37, 2843-2854.

[59] Olkhovsky, V.S. (1990) Non-stationary characteristics in study of nuclear reaction mechanism and kinetics and compound-nucleus properties. Nukleonika, 35, 99-144; (1992) Time analysis of nuclear collisions and decays. Atti Accademia Peloritana dei Pericolanti, Sci. Mat. Nat. 70, 21-40; (1998) in Mysteries, Puzzles and Paradoxes in Quantum Mechanics, Bonifacio, R. Ed., (AIP Conference Proceeding, Amer. Institute of Physics, Woodbury, NY, USA), 272-276.

[60] Olkhovsky, V.S. and Recami, E. (1992) Recent developments in the time analysis of tunnelling processes. Physics Reports, 214, 339-356; Olkhovsky, V.S., Recami, E., Raciti, F. and Zaichenko, A.K. (1995) More about tunnelling times, the dwell time and the Hartman effect. Journal de Physique, (France) I, 5, 1351-1365.

[61] Busch, Grabowski, M. and Lahti, J. (1994) Time observables in quantum theory. Physics Letters A, 191, 357-361.

[62] Kobe, D.H. and Aguilera-Navarro, V.C., (1994) Derivation of the energy-time uncertainty relation. Physical Review $A, \mathbf{5 0}, 933-938$.

[63] Blanchard, and Jadczyk, A. (1996) Timeof events in quantum theory. Helvetica Physica Acta, 69, 613-635.

[64] Grot, N., Rovelli, C. and Tate, R.S. (1996) Time of arrival in quantum mechanics. Physical Review A, 54, 4676-4690.

[65] Olkhovsky, V.S. and Agresti, A. (1997) Developments in time analysis of particle and photon tunnelling, in proceeding of the adriatico research conference on tunnelling and its implications (World Sci.; Singapore), 327-355.

[66] Olkhovsky, V.S. (1997) Time analysis of particles and photons. Physics of the Alive, 5, 19-37; (1998) Developments in examining time as a quantum-physical observable. Physics of the Alive, 6, 17-29; Olkhovsky, V. S. (1998-1999) Recent developments on time as a quantum-physical observable, Atti dell'Academia di 
Pericolanti, classe di scienze fis. mat. e natur., Universita' di Messina, v.LXXVI-LXXVII, 193-209.

[67] Leo'n, J. (1997) Time-of-arrival formalism for the relativistic particle. Journal of Physics A, 30, 4791-4801.

[68] Giannitrapani, R. (1997) Positive-operator-valued time observable in quantum mechanics. International Journal of Theoretical Physics, 36, 1575-1584.

[69] Aharonov, Y., Oppenheim, J., Popescu, S., Reznik, B. and Unruh, W. (1998) Measurement of time of arrival in quantum mechanics. Physical Review A, 57, 4130-4139.

[70] Atmanspacher, H. and Amann, A. (1998) Positiveoperator-valued measures and projection-valued measures of noncommutative time operators. International Journal of Theoretical Physics, 37, 629-660.

[71] Toller, M. (1999) Localization of events in space-time. Physical Review A, 59, 960-970.

[72] Kijowski, J. (1999) Comment on "arrival time in quantum mechanics" and "time of arrival in quantum mechanics". Physical Review A, 59, 897-899.

[73] Delgado, V. (1999) Quantum probability distribution of arrival times and probability current density. Physical Review A, 59, 1010-1020.

[74] Muga, J., Papao, J. and Leavens, C. (1999) Arrival time distributions and perfect absorption in classical and quantum mechanics. Physics Letters A, 253, 21-27.

[75] Kochànski, and Wòdkievicz, K. (1999) Operational time of arrival in quantum phase space. Physical Review A, 60, 2689-2699.

[76] Kobe, D.H., Iwamoto, H., Goto, M. and Aguilera-Navarro, V.C., (2001) Tunneling time through a barrier using the local value of a "time operator". Physical Review A, 64, Article ID 022104, 8.

[77] Muga, J., Egusquiza, I., Damborenea, J. and Delgado, V. (2002) Bounds and enhancements for negative scattering time delays. Physical Review A, 66, Article ID 042115, 8 pages.

[78] Olkhovsky, V.S., Recami, E. and Jakiel, J. (2004) Unified time analysis of photon and particle tunneling. Physical Review, 398, 133-178.

[79] Góźdź A. and Dębicki M. (2007) Time operator and quantum projection evolution. Physics of Atomic Nucle, 70, 529-536.

[80] Wang, Z.Y. and Xiong, C.D. (2007) How to introduce time operator. Annals of Physics, 322, 2304-2314.

[81] Olkhovsky, V.S. and Recami, E. (2007) Time as a quantum observable. International Journal of Modern Physics $A$, 22, 5063-5087; Olkhovsky, V.S. and Recami, E. (2008) New developments in the study of time as a quantum observable. International Journal of Modern Physics B, 22, 1877-1897.

[82] Olkhovsky, V.S. (2009) Time as a quantum observable, canonically conjugated to energy, and foundations of self-consistent time analysis of quantum processes. $A d$ vances in Theoretical and Mathematical Physics, 2009, article ID 859710, 83.

[83] Naimark, M.A. (1940) Spectral functions of a symmetric operators. Izvestiya Akademii Nauk SSSR, seriya matematicheskaya [in Russian, partially in English], 4, 277-318; we stress that it is used here the Carleman type of the spectral function of (a maximal and not only maximal ) symmetrical operator, which was cited from the reference [Carleman, T. (1923) Sur les e'quations i'ntegrales a' noyau re'el et syme'trque (Uppsala)] and was utilized as a tool of the proof, based on the approximation of the (maximal and not only maximal) symmetric operator $H$ by such succession of the bounded self-adjoint operators, the spectral functions the spectral functions of which do weakly converge to the spectral function $E(\lambda)$ of the operator $H$ (This circumstance was kindly indicated to the author by Holevo, A.S.).

[84] Aharonov, Y. and Bohm, D. (1961) Time in the quantum theory and the uncertainty relation for time and energy. Physical Review, 122, 1649-1658.

[85] Naimark, M.A. (1943) Positive definite operator functions on a commutative group, Izvestiya Akademii Nauk SSSR. seriya matematikcheskaya, 7, 237-244.

[86] Akhiezer, N.I. and Glazman, I.M. (1981) The Theory of Linear Operators in Hilbert Space, Pitman; Boston, Mass.

[87] Neuman, J. (1932) von, Mathematischen Grundlagen del Quantum Mechanik (Hizzel, Leipzig).

[88] Stone, M.H. (1930) Proceeding Nat. Acad. Sci. USA, 16, N1.

[89] ter Haar, D. (1971) Elements of hamiltonian mechanics, Oxford.

[90] Judge, D. and Levis, J.L. (1963) On the commutator $\left[L_{z}\right.$, 甲].Physics Letters, 5, 190-195.

[91] Carruthers and Nieto, M.M. (1968) Phase and angle variables in quantum mechanics. Reviews of Modern Physics, 40, 411-440.

[92] Davydov, A.S. (1976) (1982) Quantum Mechanics (Pergamon, Oxford).

[93] Chiao, R.Y., Kwiat, G. and Steinberg, A.M. (1991) Analogies between electron and photon tunneling: A proposed experiment to measure photon tunneling times. Physica B, 175, 257-262.

[94] Martin, T. and Landauer, R. (1992) Time delay of evanescent electromagnetic waves and the analogy to particle tunneling. Physical Review, A45, 2611-2617.

[95] Steinberg A.M. (1995) Conditional probabilities in quantum theory, and the tunneling time controversy. Physical Review A, 52, 32-42.

[96] Abolhasani, M. and Golshani, M. (2000) Tunneling times in the Copenhagen interpretation of quantum mechanics. Physical Review A, 62, $012106,7$.

[97] Enders, A. and Nimtz, G. (1992) On superluminal barrier traversal, Journal of Physics-I (France), 2, 1693-1698; (1993) Zero-time tunneling of evanescent mode packets. Journal of Physics-I (France), 3, 1089-1092; (1993) Photonic-tunneling experiments. Physical Review B, 47, 9605-9609; (1993) Evanescent-mode propagation and quantum tunneling. Physical Review E, 48, 632-634; G.Nimtz, in: (1997) Tunneling and its applications. World Scientific, Singapore, 223-237.

[98] Steinberg, A.M., Kwiat, G. and Chiao, R.Y. (1993) Measurement of the single-photon tunneling time. Physical Review Letter, 71, 708-711; Chiao, R.Y., Kwiat G., and Steinberg, A.M. (1993) Faster Than Light? Scient.Am., 269, 38-52; Chiao, R.Y. and Steinberg, A.M. (1997) Tunneling times and superluminality, in progress in optics, by Wolf, E., Ed., 37, Elsevier Sci., Amsterdam, 346-405.

[99] Longhi, S., et al. (2002) Measurements of superluminal optical tunneling times in double-barrier photonic band 
gaps. Physical Review E, 65, 046610.

[100] Recami, E., Olkhovsky, V.S. and Maydanyuk, S.P. (2010) On non-self-adjoint operators for observables in quantum mechanics and quantum field theory. accepted and to be published in Internat. International Journal of Modern Physics A.

[101] Macnab, R. (1978) Bacterial motility and chemotaxis molecular-biology of a behavioral system. CRC Critical Reviews in Biochemistry, 5, 291-341; Moorhead, S. and Kaplan, M.M. Ed. (1967) Mathematical challenges to the neo-darwinian interpretation of evolution. Wistar Institute Press ,Philadelphia.

[102] Behe, M.J. (1996) Darwin's Black Box. The biochemical challenge to evolution, the Free Press.

[103] Junker, R. and Scherer, S. Evolution: Ein kritisches Lehrbuch, 4th ed. Giessen (Germany): Weyel Verlag, 1998; 5th ed. Giessen (Germany): Weyel Verlag, 2001; 6th ed. Giessen (Germany), Weyel Verlag, 2006.

[104] Olkhovsky, V.S. (2001) Comparison of the faith postulates in evolutionism and creationism with respect to modern scientific data. Physics of the Alive, 9, 108-121.

[105] Prigogine, I. and Stengers, I. (1984) Order out of chaos. man's new dialogue with nature, Heinemann, London; Nicolis, G. and Prigogine, I. Exploring complexity, Freeman, W. and Co, N.Y.,1989.

[106] Prigogine, I., Nicolis, G. and Babloyants, A. (1972) Thermodynamics of evolution. Physics Today, 25, 23.

[107] Symmetries and reflections, scientific essays of Eugen
Wigner, Indiana University Press, Bloomington-London, 1970; essay 11 ("The possibility of existence of a selfreproducing system").

[108] Eigen, M. (1971) Self-organization of matter and the evolution of biological macromolecules. Naturwiss, 58, 465-523.

[109] Vol'kenstein, M.V. (1973) Physics and biology. Soviet Physics Uspekhi, 16, 207-216; see also: Vol'kenstein, M.V. (1988) Complementarity, physics and biology. Soviet Physics Uspekhi, 31, 140-150.

[110] Hartle, J.B. and Hawking, S.W. (1983) Wave function of the Universe. Physical Review D, 28, 2960-2975.

[111] Vilenkin, A. (1994) Approaches to quantum cosmology. Physical Review D, 50, 2581-2594.

[112] Kragh, H. (1996) Cosmology and controversy, Princeton (NJ), Princeton University Press.

[113] Peacock, J. (1999) Cosmological physics, Cambridge University Press.

[114] Adams, F.C. and Laughlin, G. (1997) A dying universe: the long-term fate and evolution of astrophysical objects. Reviews of Modern Physics, 69, 337-372.

[115] Carter, B. (1974) Large number coincidences and the anthropic principle in cosmology. IAU Symposium 63: Confrontation of Cosmological Theories with Observational Data, Dordrecht, Reidel.

[116] Barrow, J.D. and Tipler, F.J. (1986) The anthropic cosmological principle. Clarendon Press, Oxford. 